\title{
Evaluation of Immunochromatographic Rapid Diagnostic Test versus Peripheral Blood Smear for Diagnosis of Malaria in Nepal
}

\author{
Pande $\mathrm{PR}^{1}, \mathrm{KC} \mathrm{I}^{2}$ \\ ${ }^{1}$ Prakash Raj Pande, Senior Consultant Physician, ${ }^{2}$ Indu KC, Physician, Shree Birendra Hospital
}

\begin{abstract}
Malaria is one of the most important parasitic diseases of humans. It is the major vector disease with occasional focal outbreaks in Nepal. Annually more than 60,000 clinical cases of malaria occur out of that about 10,000 cases are confirmed cases of which about 1200 happen to be plasmodium falciparum cases. Several factors predispose to transmission of deadly / resistant strains to local population which in turn leads to cerebral malaria having both high mortality and morbidity. As it is well known that peripheral blood smear remains the "gold-standard" in diagnostic measure apart from compatible history but circumstances and situations like in periphery of developing country like ours we can not overlook the advantage of Rapid Diagnostic Tests (RDTs) like OptiMAL test which detects parasitic lactate dehydrogenase in a drop of patient's blood. This study showed that the malaria detected by OptiMAL have sensitivity $96 \%$ and specificity of $100 \%$ amongst 50 cases of malaria, the OptiMAL is able to detect 48 positive cases. Despite the cost of the test, it's sensitivity and specificity are two remarkable factors in diagnosing the disease rapidly even in case of slide/smear negative ones. The utility of RDTs are not enough studied in our part of world especially in our country where even sufficient trained manpower is always a rarity. The importance of this test emphasize the easy-to-use tool for early diagnosis and prompt institution of appropriate therapy to reduce both mortality, morbidity and in return reducing hospital stay and increasing working hour of productive manpower.
\end{abstract}

Malaria a protozoan disease transmitted by the bite of infected anopheles mosquitoes is one of the most important parasitic diseases of humans, with transmission in 103 countries affecting $\geq 1$ billion people causing between 1 and 3 million deaths each year. Malaria has now been eliminated from North America, Europe, and Russia but despite enormous control efforts, has resurged in many parts of tropics. However increasing problem of drug resistance of the parasite and insecticide resistance of the vectors in endemic areas are major concern. Malaria even today, as it has been for centuries, remains a heavy burden on tropical communities, a threat to non-endemic countries, and a danger to travelers. ${ }^{1}$ In Nepal. Every year more than 60,000 clinical cases of malaria occurs as occasional focal outbreaks out of that about 10,000 cases are confirmed cases of which about 1200 happen to be plasmodium falciparum (pf) cases. In 1990s many focal outbreak have occurred which led to some death as well. The rising incidence of malaria and the increasing resistance to treatment with conventional antimalarial drugs and the susceptibility of vectors to the available insecticides is posing challenge to and has added burden to the public health experts and scientists engaged in malaria control programme. Thus Global Malaria Control Strategy, RBMI, And National Malaria and Kala-azar control programs have set the following objective for malaria control:

Address for correspondence: prakash.pande@live.com 
Prevention of mortality due to anaemia

o Progressive reduction of malaria morbidity, endemicity, and transmission to a level that will not hinder socioeconomic development of the country

o Prevention and control of malaria epidemics

o Containment of P.falciparum epidemics

o Sustained capacity building and support control activities through operational research

o Public / private / community partrnership for improved malaria control.

Following lab tests are available to confirm malaria as per need.

o Giemsa stained PBS-Thick and Thin smear: "Gold standard"

o Acridine Orange stained PBS -[Good has some limitations\}]

o Quantitative Buffy Coat Assay - [Excellent \{has only few limitations\}]

o Rapid Diagnostic Tests: pfHRP-II and pLDH

o PCR (Polymerase Chain Reaction)

While addressing malaria in Nepal one should not forget the points including population distribution \& epidemiological changes [Terai region, Open - border with India] and security troops [contribution of troops from Nepalese Army, Police and Armed -forces in peace keeping mission in endemic parts of African continent] endemic area visit.

Along with several other factors those aforementioned factors are again predisposing to the transmission of deadly / resistant strains to local population which in turn leads to cerebral malaria having both high mortality and morbidity. RDTs use should be evaluated for early diagnosis and prompt institution of therapy is an important determinant of outcome especially in case of severe falciparum malaria. Important factors to be considered while opting RDT'S are; (i) commercial availability (ii) Plasmodium species to be detected (p.falciparum / pan-specific), (iii) cost (including transport, training, and quality control), (iv sensitivity, (v). shelf-life and temperature stability in intended conditions of storage and use, (vi) ease of use including format of the test (e.g. cassette, dipstick, car) and (vii) requirement for post -treatment testing of patient.

This study was carried out with pLDH based Rapid Diagnostic Tests (RDTs) to be evaluated in diagnosis of malaria, probable / suspected malaria cases visiting Shree Birendra Hospital. As it is well known that peripheral blood smear remains the" gold- standard" in diagnostic measure apart from compatible history but circumstances and situations like in periphery of developing country like ours we can not overlook the advantage of Rapid Diagnostic Tests (RDTs) like Opti MAL test. Despite the cost its sensitivity and specificity are two remarkable factors in diagnosing the disease rapidly even in case of situations like slide/smear negative ones. Even the sporadic endemic have great toll in the health cost of developing countries like ours. Presentation of disease is usually similar to Enteric fever or even hepatitis which not only imparts diagnosticdilemma but also delays in institution of therapy timely to eradicate the parasite from blood. This results increase in number of hospital stay and thereby delaying their recovery and ultimately reducing the working hours. As implication of rapid tests/ RDTs are not enough studied in our part of world especially in our country where sufficient trained manpower in technical field is always a rarity! At times availability of microscopy and electricity may not only be impossible but also time consuming and cumbersome procedure in endemic situations. We need to develop skill and keep us standby with the easy alternative method to tackle such cases in emergency and unconventional situations without hurdles and promptly.

\section{Subject and Methodology}

This was a Hospital based descriptive case study carried out at Medical and Pathology department of Shree Birendra Hospital, Chhauni, Ktm, involving the sample Size 50- case of high- grade fever with history of travel to endemic area and with or without malaria prophylaxis. As this hospital covers patients/army personnel from various parts of Nepal including Terai region where the prevalence of malaria is high. Further, the Nepalese Army personnel also travel overseas to serve in the United Nations missions including that in Africa and malaria is severe there as well. This study also involved any patients / army personnel serving in malaria endemic zone inside Nepal and person with travel history to malaria endemic zone in Africa as part of service to UN peacekeeping missions. Patients with history of high-grade fever clinically diagnosed as a case of malaria were included after taking informed 
consent. Thorough evaluation was done including obtaining a detailed history, physical examination and laboratory investigations (as in Performa) of all enrolled patients. Demographic variables included age, sex, marital status and occupation of the patients. History of travel to endemic area, presence or absence of malaria prophylaxis, presence or absence of jaundice, past history of blood transfusion, past malaria and treatment history was taken with emphasize to radical curative use in indicated cases. A detailed clinical examination was done with emphasis on temperature pattern its relation with pulse, BP, respiration, pallor / icterus (jaundice), abdominal examination for any tenderness and presence of hepatomegaly/ splenomegaly. Anthropometiric measurements including height, weights were taken and BMI was calculated.

All the samples were collected by the residents/ technicians in sterile vials containing EDTA as anticoagulant and processed immediately in parasitology laboratory, Department of Microbiology/ Pathology, Birendra Army Hospital. Routine and specific laboratory tests were carried out within 1 to $2 \mathrm{hrs}$ of collection of sample using standard laboratory protocol. Few drop of blood was taken out from the finger pulp after wiping it with spirit swab by using sterile disposable surgical blade and samples were kept in two separate vials and two glass slides. Thin blood film was prepared for species identification.

* $\quad$ CBC comprising TC, DC, Hemoglobin, Haematocrit, Platelets, and ESR was done.

* $\quad$ Few drops of blood (2-3drops) were kept in two separate glass-slide for carrying out the microscopic examination of blood smears for about 3minute each[ (a) Thin smear \&(b) Thick smear].

Immunochromatological test using OptiMAL assay - kit.

OptiMAL test is designed to detect infections with both $P$ falciparum/ $P$ vivax. Manufacturer's instructions were followed strictly in both tests. Only a single drop (about 30ul) of the fresh whole blood was used for this test. Methodology was as follows:

1. One drop of the buffer solution was added to the conjugated well and similarly four drops use was added to the second test well.

2. One drop (about 10ul) of blood obtained by finger prick was added to the conjugate well with the help of capillary tube provided in the kit and mixed gently.
3. The dipstick was then placed in conjugate well with its wick at the top for 10 minute.

4. After $10 \mathrm{~min}$, the test strip was transferred to the second well which contained the clearing buffer and kept for about $5 \mathrm{~min}$.

5. Then, the dipstick was removed from the well and the interpretation was made after observing the result.

6. In the OptiMAL assay, the reaction is visualized in the form of pink bands across the strip. There are 3 reaction zones in the OptiMAL dipstick.

- A mono-specific antibody that recognizes only $P$ falciparum is coated in the bottom reaction line.

- A second pan specific antibody is immediately above this zone. This monoclonal antibody that recognizes the pLDH informs of $P$ vivax.

- A third reaction zone is coated at the top of the dipstick where there is an antibody that captures the excess colloid conjugate and serves as a positive control for assay.

The reaction bands are seen as pink-mauve line in the test strip. The interpretation of the assay test strip results is as follows:

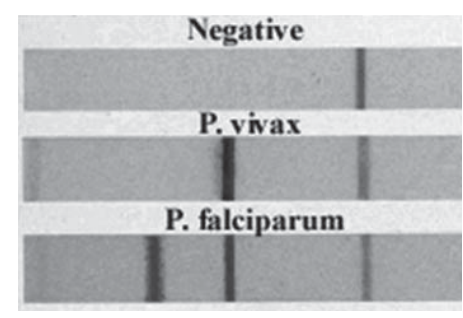

Negative (one control band at the top)

Positive for $\mathrm{P}$ vivax (one control band plus one test band)

Positive for $P$ falciparum (one control band plus two test band).

\section{Observations \& Results}

All the specimens were examined within 2-6 hours of collections. Giemsa stained slides were reported negative only after examination of 200-300 fields systematically. Positive blood films were recorded as Parasites (Rings/ Trophozoites / Schizonts) per 100 white blood cells. [Microscopic demonstration of any morphological stages of malaria parasite was considered as the sole criteria (Gold standard) for laboratory 
diagnosis of malaria.]All the samples were examined by using both techniques for the laboratory diagnosis of malaria using standard protocols. Results were noted as M. P.- Positive(+)Stage. Species- Rings/ Schizont / Trophozoite / Gamete of P.vivax / falciparum. Out of 50 patients 48 were positive for optimal test while 2 were negative and out of that 12 were p. falciparum and 36 were p.vivax and 1 each negative for both species.

\section{Discussion}

The discovery of malaria parasites by Laveran in 1880 added milestone in the history of malaria. Malaria is traditionally been diagnosed by examining Giemsa stained blood films, which is considered as the 'gold-standard' method around the world and also in Nepal. In our study we carried out both PBS and RDTs for their comparision. This study showed that the malaria detected by OptiMAL have sensitivity $96 \%$ and specificity of $100 \%$ amongst 50 cases of malaria the OptiMAL is able to detect 48 positive cases. The 2 blood samples (the positive) were not detected by the OptiMAL test as positive as these patients had taken anti-malarial chemotherapy which might have already killed the parasites and only dead parasites might have been appeared in the slides. The reason would be that OptiMAL assay had not detected them as it is well known that OptiMAL assay captures the pLDH antigen which was secreted only by live organisms. The sensitivity of the OptiMAL for detection of $P$ vivax was $97.3 \%$ and for detection of $P$ falciparum was $92.3 \%$. The blood film could only detect and indicated that 70\%(35 of 50) of the patients of which $77.14 \%$ (27 of 35) were positive for $\mathrm{P}$ vivax and $22.9 \%$ (8 of 35) were positive for $\mathrm{P}$ falciparum. Our data was very much compatible with the results from Honduras i.e. sensitivity of $94 \%$ and specificity of $100 \%$ for Opti MAL assay ${ }^{2,3}$. Another study conducted in University of Munich, Germany in 1998, similar results were obtained for Opti MAL assay, with a sensitivity of $88.7 \%$ \& a specificity of $91.9 \%$. Similar study was carried out by Prakash Ghimire (2004) in three holoendemic districts in Far western regional VDCs of Kanchanpur, southern Nepal (Jhalari, Rampur-Bisalpur \& Krishnapur). The result obtained was sensitivity of $85 \%$ and specificity $100 \%$.,PPV $100 \%$ \& NPV of $89.28 \% \%^{4}$. In another study carried out by Sherchand JB (2001) in the endemic districts of Nepal the result obtained was having the sensitivity of $97 \%$ and specificity of $98 \%$ for OptiMAL assay ${ }^{5}$. Grobusch MP, et al carried out a study with interesting result that detection of False-positive rapid tests was high for malaria in patients with presence of rheumatoid factor ${ }^{6}$. It is also true that PCR test for malaria has high yield in cases where mixed inection is suspected because RDTs cannot differentiate species in case of mixed infection. Snounou G., et al. studied Identification of the four human malaria parasite species in field samples by the polymerase chain reaction and detection of a high prevalence of mixed infections ${ }^{7}$.

In conclusion, RDTs have introduced a new dimension to the diagnosis and treatment of malaria. They now permit, among other things, on-the-spot confirmatory diagnosis of malaria at the periphery of the health care system, by health workers with minimal training. The OptiMAL test has thus special significance in a country like Nepal where most of rural centers are unable to diagnose malaria due to the lack of microscopes even slides for microscopy, stains and trained technicians. Malaria endemic zones in Nepal are usually in rural villages, which are at far distance from the Highways. Due to the poor economic condition, distant health centers, lack of transportation facility, patients cannot go to the health centers for treatment thus increasing mortality. In these group of patients follow up is also very difficult. Therefore prompt diagnosis and treatment is a must for the rural malaria patients. It can be accomplished by the use of OptiMAL test. The test is extremely simple and rapid to perform, making it easy to teach the technique/methodology to inexperienced or even untrained persons. Besides the financial savings from unnecessary treatments, the use of OptiMAL test is of value in the early investigations and management of malarial endemics. The rational use of RDTs as a complement to microscopy might give substantial health benefits through earlier treatment and a consequent reduction in morbidity and mortality, by targeting expensive drugs and drug combinations to high risk populations in multidrug resistant areas and through a more rational use of drugs that might effectively reduce drug pressure and possibly delay the progress of drug resistance. The OptiMAL test is of great value in diagnosis of severe and complicated falciparum malaria when microscopy testing might delay institution of treatment promptly. In addition, presence of pLDH in blood indicates presence of viable malaria parasites; the test can be used to evaluate the effectiveness of antimalarial chemotherapy. It is thus a simple way to monitor response to the drug therapy and in the detection of drug resistance Malarial strains because pLDH reflects the presence of viable Malaria parasites in the blood. pLDH levels follow closely parasitism. Nevertheless, RDTs are unlikely to be widely adopted until their detection capacities have been improved, their potential benefits have been confirmed, and their cost has come closer to what most national malaria programme can afford. 
To address these issues, and ensure the optimal use of RDTs as a key tool in malaria control, it requires a co-ordinated effort among users, control programmes, manufacturers and international agencies.

\section{Acknowledgements}

We are especially thankful to ward staff and laboratory technologists of Shree Birendra Hospital, Chhauni who worked with us on this study.

\section{References}

1. Nicholas J. White, Joel G Breman, Malaria, Harrison's principles of Internal Medicine vol I, $16^{\text {th }}$ edition, Mc Graw Hill 2005 p1218-1233.

2. Makler M T, Palmer C J and Ager A.L.;A review of practical techniques for diagnosis of malaria. Ann. Trop.Med \& Parasitol.1998, 92(4),419-433.

3. Quintana M., R Piper, H.L. Boling, Mmarker, C Sherman, E Gill, F Fernandez,S Marten: Malaria Diagnosis in Honduran population with coindemic P. falciparum and P. vivax. Am.J.Trop.Med. Hyg.1998,59(6)868-871.
4. Ghimire Prakash, Puspa Raj Pande,Deepak Joshi Yogan Khatri, Keshab Parajuli.evaluation of rapid immunochromatographic test for diagnosis of malaria in Nepal. Scientific World, HMG Nepal2004;2(2):40-44.

5. Sherchand JB, Rapid immunochromatographic OptiMAL assay for detection of $P$ vivax and $P$ falciparum malaria from two districts of Nepal, Nepalese Journal of Medical Laboratory Sciences, 2001;1:21-25.

6. Grobusch MP, et al. False-positive rapid tests for malaria in patients with rheumatoid factor. Lancet, 1999, 353:297.

7. Snounou G, et al. Identification of the four human malaria parasite species in field samples by the polymerase chain reaction and detection of a high prevalence of mixed infections. Molecular and Biochemical Parasitology,1993, 58:283-292. 